\title{
Corrigendum
}

\section{Paradoxical effects of short-term antidepressant treatment in fMRI emotional processing models in volunteers with high neuroticism - CORRIGENDUM}

\author{
M. Di Simplicio, R. Norbury, A. Reinecke and C. J. Harmer \\ doi:10.1017/S0033291713000739. Published online by Cambridge University Press, 19 April 2013.
}

In the Introduction to this paper (Di Simplicio et al. 2013) the authors incorrectly refer to Bigos et al. (2008) among a number of previous studies consistently showing a decreased amygdala response to negative versus positive facial expressions after single-dose antidepressant administration in unselected healthy volunteers. Contrary to the other findings cited, Bigos et al. (2008) found an increased amygdala response to facial expressions after acute citalopram administration.

Instead, Bigos et al. (2008) should have been cited in the Limitations paragraph, a corrected version of which is displayed below.

\section{Limitations}

The results from this study are limited by the absence of a low-N control group also receiving SSRI treatment to verify that the effects seen here are specific to a highly neurotic sample. However, early effects of SSRI treatment on unselected (typically low-anxious) populations have been well replicated, with one exception using high-dose citalopram (20 mg i.v. compared to $20 \mathrm{mg}$ oral administration) (Bigos et al. 2008). We also did not take a measure of drug plasma levels to ensure compliance and it is possible that the volunteers in this study did not all take citalopram as instructed, even though clear differences were apparent compared to placebo. Finally, in the absence of post-scan questionnaires verifying whether subjects were able to refrain from focusing on their thoughts during the ASL resting sequence, it possible that the two treatment groups engaged in different mental activities that might have been reflected in the amygdala perfusion differences.

\section{References}

Bigos KL, Pollock BG, Aizenstein HJ, Fisher PM, Bies RR, Hariri AR (2008). Acute 5-HT reuptake blockade potentiates human amygdala reactivity. Neuropsychopharmacology 33, 3221-3225.

Di Simplicio M, Norbury R, Reinecke A, Harmer CJ (2013). Paradoxical effects of short-term antidepressant treatment in fMRI emotional processing models in volunteers with high neuroticism. Psychological Medicine. doi:10.1017/S0033291713000739. 